\title{
THE COBE DIRBE SEARCH FOR THE COSMIC INFRARED BACKGROUND
}

\author{
MICHAEL G. HAUSER \\ Code 680, Laboratory for Astronomy and Solar Physics \\ NASA Goddard Space Flight Center, Greenbelt, MD 20771 \\ $U S A$
}

\begin{abstract}
The Diffuse Infrared Background Experiment (DIRBE) on the Cosmic Background Explorer ( $C O B E$ ) satellite is designed to conduct a sensitive search for isotropic cosmic infrared background radiation over the spectral range from 1.25 to $240 \mu \mathrm{m}$. The cumulative emissions of pregalactic, protogalactic, and evolving galactic systems are expected to be recorded in this background. The DIRBE instrument has mapped the full sky with high redundancy at solar elongation angles ranging from $64^{\circ}$ to $124^{\circ}$ to facilitate separation of interplanetary, Galactic, and extragalactic sources of emission. Conservative limits on the isotropic infrared background are given by the minimum observed sky brightnesses in each DIRBE spectral band during the 10 months of cryogenic operation. Extensive modeling of the foregrounds is under way to isolate or strongly limit the extragalactic infrared component. The current approach to these modeling efforts is described and representative present residuals are reported.
\end{abstract}

\section{Introduction}

The search for cosmic infrared background (CIB) radiation is a relatively new field of observational cosmology. Measurement of this distinct radiative background, expected to arise from the cumulative emissions of pregalactic, protogalactic, and galactic systems, would provide new insight into the cosmic 'dark ages' following the decoupling of matter from the cosmic microwave background radiation (Partridge \& Peebles 1967; Low \& Tucker 1968; Peebles 1969; Harwit 1970; Kaufman 1976; Bond, Carr, \& Hogan 1986, 1991; Franceschini et al. 1991; Franceschini et al. 1994). Observation- 
ally, there have been no corroborated direct detections of the CIB, though possible evidence for an isotropic infrared background, or at least limits on emission in excess of galactic foregrounds, has been reported in data from rocket experiments (Matsumoto et al. 1988; Matsumoto 1990; Noda et al. 1992; Kawada et al. 1994). Stringent limits have been set on any extragalactic background from 500 to $5000 \mu \mathrm{m}$ wavelength in excess of that in the cosmic microwave background (CMB) radiation (Mather et al. 1994; Wright et al. 1994). The status of direct attempts to detect the CIB was recently reviewed by Hauser (1995). Indirect limits on the extragalactic infrared background have been inferred from possible attenuation of $\mathrm{TeV}$ $\gamma$-rays due to pair-production by photon-photon interactions (De Jager, Stecker, \& Salamon 1994; Dwek \& Slavin 1994; Biller et al. 1995).

The Diffuse Infrared Background Experiment (DIRBE) on the COBE spacecraft is the first satellite instrument designed specifically to carry out a systematic search for the CIB. The COBE FIRAS instrument, designed primarily to make a precise measurement of the spectrum of the cosmic microwave background radiation, is also a powerful instrument for the CIB search in the submillimeter range (Mather, this Symposium). The search for the CIB is the most exploratory of the $C O B E$ objectives. Even from a spaceborne instrument, this cosmic fossil is far more difficult to discern than the CMB radiation. Whereas the CMB is the dominant celestial radiation over most of the sky at millimeter wavelengths, the local infrared foregrounds from interplanetary dust and the Galaxy are far brighter than the CIB.

Conservative upper limits on the CIB based on preliminary reduction of DIRBE measurements of the south ecliptic pole brightness, and a comparison of DIRBE data with those from instruments on sounding rockets and the Infrared Astronomical Satellite (IRAS), were presented by Hauser et al. (1991). The data collection phase of the DIRBE investigation is now completed, and the data from the period of cryogenic operation of the instrument (18 Nov 89 to 21 Sept 90 ) are fully reduced to calibrated sky maps. In this paper we report updated CIB limits in the DIRBE spectral range based upon the faintest sky brightnesses observed at each wavelength and a more recent calibration of the DIRBE data than that used by Hauser et al. (1991). We also describe the initial modeling of the solar system and Galaxy foreground contributions to the infrared sky brightness by the DIRBE team, and indicate the nature of the residuals.

\section{The Observational Challenge}

The search for the CIB is impeded by two fundamental challenges: there is no unique spectral signature of such a background, and there are many 
local contributors to the infrared sky brightness at all wavelengths, often quite bright. The lack of a distinct spectral signature arises in part because so many different sources of primordial luminosity are possible (e.g., Bond, Carr, \& Hogan 1986), and in part because the primary emissions are then shifted into the infrared by the cosmic red-shift and dust absorption and re-emission. Hence, the present spectrum depends in a complex way on the characteristics of the luminosity sources, on their cosmic history, and on the dust formation history of the Universe.

Setting aside the difficult possibility of recognizing the CIB by its angular fluctuation spectrum (Bond, Carr, \& Hogan 1991), the only identifying CIB characteristic for which one can search is an isotropic glow. One must solve the formidable observational problem of making absolute brightness measurements in the infrared. One must then discriminate and remove the strong signals from foregrounds arising from one's instrument or observing environment, the terrestrial atmosphere, the solar system, and the Galaxy. Particular attention must be given, of course, to possible isotropic contributions from any of these foreground sources. Finally, if an extragalactic isotropic residual remains, one must evaluate the contribution from galaxies over their luminous lifetime to distinguish their light from that of pregalactic or protogalactic sources. The DIRBE investigation is presently at the stage of modeling the known solar system and Galactic foregrounds and assessing the uncertainties in the models and measurements so as to determine whether evidence for an isotropic residual remains.

\section{The COBE Diffuse Infrared Background Experiment}

The primary aim of the Diffuse Infrared Background Experiment (DIRBE) is to conduct a definitive search for an isotropic CIB, within the constraints imposed by the local astrophysical foregrounds. The experimental approach was to obtain absolute brightness maps of the full sky in 10 photometric bands at $1.25,2.2,3.5,4.9,12,25,60,100,140$, and $240 \mu \mathrm{m}$. In order to facilitate discrimination of the bright foreground contribution from interplanetary dust, linear polarization was also measured at $1.25,2.2$, and 3.5 $\mu \mathrm{m}$. Because of the Earth's motion within the interplanetary dust cloud, the diffuse infrared brightness of the entire sky varies over the course of a year. To monitor this variation, the DIRBE field of view was offset from the $C O B E$ spacecraft spin axis by $30^{\circ}$. This produced a helical scan of the sky during each $C O B E$ orbit. Over the course of six months this yielded observations of each celestial direction hundreds of times at all accessible solar elongation angles (depending upon ecliptic latitude) in the range $64^{\circ}$ to $124^{\circ}$. All spectral bands viewed the same instantaneous field-of-view, and the helical scan allowed the DIRBE to map $50 \%$ of the celestial sphere 
each day. The instrument was designed to achieve a sensitivity at each wavelength for each $0.7^{\circ} \times 0.7^{\circ}$ field of view of approximately $\nu I_{\nu}=1 \mathrm{nW}$ $\mathrm{m}^{-2} \mathbf{s r}^{-1}(1 \sigma, 1$ year$)$. This is well below the actual sky brightness, and below many of the predictions for the CIB, at all wavelengths.

The DIRBE instrument is an absolute radiometer, utilizing an off-axis folded Gregorian telescope with a 19-cm diameter primary mirror. The optical configuration (Magner 1987) was carefully designed for strong rejection of stray light from the Sun, Earth limb, Moon or other off-axis celestial radiation, or parts of the $C O B E$ payload (Evans 1983). The instrument, which was maintained at a temperature below $2 \mathrm{~K}$ within the $C O B E$ superfluid helium dewar, measured absolute brightness by chopping between the sky signal and a zero-flux internal reference at $32 \mathrm{~Hz}$. Instrumental offsets were measured about five times per orbit by closing a cold shutter located at the prime focus. A radiative offset signal in the long wavelength detectors arising from JFETs (operating at about $70 \mathrm{~K}$ ) used to amplify the detector signals was identified and measured in this fashion. Internal radiative reference sources were used to stimulate all detectors when the shutter was closed to monitor the stability and linearity of the instrument response. The highly redundant sky sampling and frequent response checks provided precise photometric closure over the sky and reproducible photometry to $\sim$ $1 \%$ or better for the duration of the mission. Calibration of the photometric scale was obtained from observations of isolated bright celestial sources. Comparison of the DIRBE and FIRAS absolute calibrations at 100, 140, and $240 \mu \mathrm{m}$ shows excellent agreement between these two independent instruments on $C O B E$. A more detailed description of the $C O B E$ mission has been given by Boggess et al. (1992), and the DIRBE instrument has been described by Silverberg et al. (1993).

\section{The Character of the Infrared Sky}

The DIRBE sky brightness maps show the dominant anticipated features of Galactic starlight and zodiacal light at short wavelengths, emission from the interplanetary dust dominating at 12 and $25 \mu \mathrm{m}$, and emission from the interstellar medium dominating at longer wavelengths (Hauser 1993; DIRBE Sky Maps at Elongation $90^{\circ}$, available from the National Space Science Data Center). The brightness of the sky in any fixed direction varies roughly sinusoidally over the year, though the detailed time dependence and amplitude of variation depends upon direction in the sky (Kelsall et al. 1993), indicating rather complex features of the interplanetary dust cloud. The DIRBE polarization maps reveal the expected strong linear polarization arising from the zodiacal light (Berriman et al. 1994). These data dramatically illustrate both the challenge of distinguishing the CIB from 
signals arising in our local cosmic environment, and the value of the DIRBE data for studies of the interplanetary medium and the Galaxy.

The most credible direct observational limits on the CIB are the minimum observed sky brightnesses. Since publication of the spectrum of a nominally dark direction, the south ecliptic pole, which was based on a quick-look reduction and initial calibration of the DIRBE data (Hauser et al. 1991), the complete data set acquired while the DIRBE was cryogenically cooled has been processed with an improved calibration. In each DIRBE weekly sky map, the faintest direction was determined for each wavelength. The smallest of these values at each wavelength over the duration of the mission provides a refined 'dark sky' limit to the CIB. Table 1 lists these dark sky values. At wavelengths where interplanetary dust scattering or emission is strong, the sky is darkest near the ecliptic poles. At wavelengths where the interplanetary cloud signal is rather weak (i.e., longward of $100 \mu \mathrm{m}$ ), the sky is darkest near the galactic poles or in minima of HI column density. The error shown for each value is the present absolute calibration uncertainty.

TABLE 1. UPPER LIMITS ON THE COSMIC INFRARED BACKGROUND

\begin{tabular}{|c|c|c|}
\hline$\lambda$ & $\begin{array}{c}\nu I_{\nu} \\
\mu \mathrm{m}\end{array}$ & Reference \\
\hline 1.25 & $393 \pm 13$ & DIRBE dark sky \\
2.2 & $150 \pm 5$ & $"$ \\
3.5 & $63 \pm 3$ & $"$ \\
4.9 & $192 \pm 7$ & $"$ \\
12 & $2660 \pm 310$ & $"$ \\
25 & $2160 \pm 330$ & $"$ \\
60 & $261 \pm 22$ & $"$ \\
100 & $74 \pm 10$ & $"$ \\
140 & $57 \pm 6$ & $"$ \\
240 & $22 \pm 2$ & \\
& & \\
$500-5000$ & $680 / \lambda(\mu \mathrm{m})$ & Mather et al. (1994) \\
\hline
\end{tabular}

As described by Mather et al. (1994), the CMB spectrum in the wavelength range $0.5-5 \mathrm{~mm}$ deviates from a $2.726 \mathrm{~K}$ blackbody shape by less than $\mathbf{0 . 0 3 \%}$ of the peak intensity. Taking this as an upper limit to an additional cosmic infrared background implies $\nu I_{\nu}<340 / \lambda(\mu \mathrm{m}) \mathrm{nW} \mathrm{m}^{-2} \mathrm{sr}^{-1}$. To make some allowance for systematic errors in separating the Galactic 
signal from the FIRAS CMB signal, the above limit has been doubled in Table 1.

\section{Discriminating the Infrared Foregrounds}

In order to determine whether an isotropic signal is present, thus measuring or setting more stringent limits upon the CIB, one must address the problem of discriminating the various contributions to the measured sky brightness. The DIRBE team is presently approaching this in a sequential process: (1) find a parameterized model of the interplanetary dust (IPD) cloud; (2) integrate this IPD model along the line of sight to calculate the sky brightness for each celestial direction for each week of the DIRBE mission; (3) subtract the calculated IPD map from each weekly DIRBE map and average the residual weekly maps; (4) blank bright sources discernible in the residual averaged maps, both stellar and more extended sources; (5) use a detailed model of discrete Galactic sources to calculate a map of the integrated sky brightness due to sources fainter than those blanked in the previous step, and subtract this map from the DIRBE residual map; (6) determine an interstellar medium emission map at each wavelength using the DIRBE $100 \mu \mathrm{m}$ map as a template, and subtract this map from the residual map; (7) test the final residual maps for isotropy.

The initial interplanetary dust model is very similar to that used by Good in creating the IRAS Sky Survey Atlas (Wheelock et al. 1994). The cloud is assumed to have an intrinsically static, Sun-centered density distribution, with a symmetry plane inclined to the ecliptic plane. The combined effects of symmetry plane inclination and eccentricity of the Earth's orbit produce the annual variations of sky brightness in the model. The model is extended to the short-wavelength DIRBE bands by introducing particle albedos and phase functions which are sums of Henyey-Greenstein functions. Our first test model includes only a main 'smooth' IPD cloud. The IPD dust bands discovered in the IRAS data (Low et al. 1984) and confirmed in the DIRBE data (Spiesman et al. 1995) and the dust ring containing particles resonantly trapped just outside $1 \mathrm{~A}$. U. (Dermott et al. 1994; Reach et al. 1995) will be incorporated in future versions. The polarization data, and corresponding parameters to characterize particle properties, are also ignored in the initial model. The 14 free parameters of the model are determined by simultaneously fitting the time variation of the sky brightness at several hundred lines of sight over the 10 months of cryogenic operation. Though this simple model represents the IPD signal fairly well, typically to several percent of the brightness in the ecliptic plane, there are clearly systematic artifacts in the residuals which can be reduced by more refined models. The model shows that the IPD signal is a large fraction of the sky 
brightness at wavelengths short of $100 \mu \mathrm{m}$, even at high galactic latitude. The uncertainty in the residual sky maps at these wavelengths will be dominated by uncertainties in the IPD signal removal until more refined models are developed.

The sky brightness contribution from faint Galactic sources is obtained by integrating the source counts in the model of Wainscoat et al. (1992), as elaborated by Cohen $(1993 ; 1994)$. The model includes 87 types of Galactic sources, distributed in spatial components including the disk, bulge, halo, spiral arms, and the molecular ring. Galactic extinction is included in the model. Sky brightnesses in each DIRBE band from 1.25 to $25 \mu \mathrm{m}$ were calculated for 238 sky zones (M. Cohen, private communication to the DIRBE team). At high galactic latitudes these sources contribute $5-20 \%$ of the observed sky brightness from 1.25 to $4.9 \mu \mathrm{m}$; at 12 and $25 \mu \mathrm{m}$ their contribution is less than $0.1 \%$.

The initial model of interstellar medium (ISM) emission assumes uniform color, at least at high galactic latitude. The $100 \mu \mathrm{m}$ DIRBE residual map is used as a spatial template for the ISM because of its high signalto-noise ratio. The color for each band is determined from the slope of the correlation between the brightness in that band and the $100 \mu \mathrm{m}$ brightness. The $100 \mu \mathrm{m}$ brightness not associated with any apparent ISM material is found by correlating $100 \mu \mathrm{m}$ brightness at high latitude with $21-\mathrm{cm} \mathrm{HI}$ emission (Stark et al. 1992), and extrapolating this correlation to zero 21$\mathrm{cm}$ emission. This assumes either that HI gas is a good spatial tracer of all interstellar gas, or that infrared emission from other gas phase components is negligible. Tests made using available high latitude CO and HII maps show that this is a reasonable first approximation. This method for estimating the ISM contribution has been used for wavelengths of $12 \mu \mathrm{m}$ and longer. A modified procedure which distinguishes extinction and emission effects is under study for determining the shorter wavelength ISM contribution. Rough estimates suggest that the ISM signal at the short wavelengths is small compared to the present uncertainties in the IPD model. At wavelengths of 140 and $240 \mu \mathrm{m}$ the ISM signal is the largest foreground contribution to the sky brightness at high latitudes.

\section{Status of the CIB Search}

The process of refining the DIRBE foreground models, assessing the uncertainties in the models and the measurements, and testing the residuals for isotropy is not yet complete. Hence, no conclusions regarding DIRBE detection of, or limits upon, the CIB more restrictive than the dark sky limits in Table 1 can yet be stated.

Some insight into the present status is provided by looking at the resid- 
ual brightnesses in several high latitude regions. For this purpose, we have examined the residuals in $10^{\circ} \times 10^{\circ}$ fields at the Galactic poles and ecliptic poles, and a $5^{\circ} \times 5^{\circ}$ field in the 'Lockman Hole' (Lockman, Jahoda, \& McCammon 1986; Jahoda, Lockman, \& McCammon 1990), the region of minimum HI column density at $(\ell, b) \sim\left(148^{\circ},+53^{\circ}\right)$. Table 2 lists the minimum and maximum mean residual brightnesses for these 5 patches after all of the foreground removal steps described above. While the range of values at each wavelength is still substantial, typically a factor of 2 or more, comparison with Table 1 shows that we are now dealing with residuals which are small fractions, approaching $10 \%$ at wavelengths shortward of $100 \mu \mathrm{m}$, of the 'dark sky' limits. However, the fact that the residuals are brightest in the region of peak IPD thermal emission, 12 to $25 \mu \mathrm{m}$, strongly suggests that significant foreground emission still remains, at least in the middle of the DIRBE spectral range.

\section{TABLE 2. RANGE \\ OF SKY BRIGHTNESS \\ RESIDUALS AT HIGH LATITUDE}

\begin{tabular}{|c|c|}
\hline $\begin{array}{c}\lambda \\
\mu \mathrm{m}\end{array}$ & $\begin{array}{c}\nu I_{\nu} \\
\left(\mathrm{nW} \mathrm{m}^{-2} \mathrm{sr}^{-1}\right.\end{array}$ \\
\hline 1.25 & $59-164$ \\
2.2 & $15-71$ \\
3.5 & $9-25$ \\
4.9 & $15-42$ \\
12 & $104-330$ \\
25 & $121-287$ \\
60 & $27-45$ \\
100 & $23-41$ \\
140 & $24-50$ \\
240 & $12-25$ \\
\hline
\end{tabular}

As a complement to the direct searches for the CIB by diffuse infrared background measurements, galaxy counts in the infrared allow estimation of lower limits to the total extragalactic infrared background. For example, Cowie et al. (1990) estimated the integrated contribution of galaxies at 2.2 $\mu \mathrm{m}$ to be $\nu I_{\nu}=5 \mathrm{nW} \mathrm{m}^{-2} \mathrm{sr}^{-1}$ on the basis of deep galaxy counts. Hacking \& Soifer (1991) used galaxy luminosity functions derived from IRAS data to predict minimum backgrounds (integrated to $z=3$ ) at 25,60 , and 100 $\mu \mathrm{m}$ of 1,2 , and $4 \mathrm{nW} \mathrm{m} \mathrm{m}^{-2} \mathrm{sr}^{-1}$ respectively. Beichman \& Helou (1991) 
used synthesized galaxy spectra, also based largely on IRAS data, to estimate the diffuse infrared background due to galaxies. At $300 \mu \mathrm{m}$, their minimum estimated brightness (integrated to $z=3$ ) is $2 \mathrm{nW} \mathrm{m} \mathrm{m}^{-2} \mathrm{sr}^{-1}$. The integrated galaxy far-infrared background contribution may exceed these estimates substantially if there has been evolution in galaxy luminosity or space density: deeper counts from future space infrared observatories such as $I S O$ and SIRTF will improve these estimates. Even these minimum extragalactic background contributions should be detectable if the foreground contributions to the $C O B E$ measurements can be modeled to about the $1 \%$ level, a difficult but perhaps achievable goal.

\section{Conclusion}

Measurement of the extragalactic infrared background radiation will advance our understanding of cosmic evolution since decoupling. The high quality and extensive new measurements of absolute infrared sky brightness obtained with the DIRBE and FIRAS instruments on the COBE mission have already set significant limits upon this elusive background.

When foreground models are completed and uncertainties carefully assessed, the $C O B E$ data will certainly provide cosmologically valuable constraints upon, or perhaps even direct detection of, the extragalactic infrared background to the limits imposed by our bright astrophysical environment.

\section{Acknowledgments}

The author gratefully acknowledges the contributions to this report by the many participants in the $C O B E$ Project, to his colleagues on the $C O B E$ Science Working Group, and especially the many scientists, analysts, and programmers engaged in the DIRBE investigation. Those particularly engaged in the work reported here include E. Dwek, T. Kelsall, H. Moseley, R. Silverberg, E. Wright, R. Arendt, G. Berriman, B. Franz, H. Freudenreich, C. Lisse, M. Mitra, N. Odegard, W. Reach, J. A. Skard, T. Sodroski, S. Stemwedel, G. Toller, and J. Weiland. The National Aeronautics and Space Administration/Goddard Space Flight Center (NASA/GSFC) is responsible for the design, development, and operation of the $C O B E$. Scientific guidance is provided by the $C O B E$ Science Working Group. GSFC is also responsible for the development of the analysis software and for the production of the mission data sets.

\section{References}

Beichman, C. A. \& Helou, G., 1991, ApJ, 370, L1

Berriman, G. B., et al. , 1994, $A p J, 431$, L63 
Biller, S. D., et al. , 1995, ApJ, to be published

Boggess, N. et al. , 1992, ApJ, 397, 420

Bond, J. R., Carr, B. J., \& Hogan, C. J., 1986, ApJ, 306, 428

Bond, J. R., Carr, B. J., \& Hogan, C. J., 1991, ApJ, 367, 420

Cohen, M., 1993, $A J, 105,1860$

Cohen, M., 1994, $A J$, 107, 582

Cowie, L. L., et al. , 1990, ApJ, 360, L1

De Jager, O. C., Stecker, F. W. \& Salamon, M. H., 1994, Nature, 369, 294

Dermott, S. F. et al. , 1994, Nature, 369, 719

Dwek, E. \& Slavin, J., 1994, ApJ, 436, 696

Evans, D. C., 1983, SPIE Proc., 384, 82

Franceschini, A. et al. , 1991, A\&A SupplSer, 89, 285

Franceschini, A. et al. , 1994, $A p J, 427,140$

Hacking, P. B. \& Soifer, B. T., 1991, ApJ, 367, L49

Harwit, M., 1970, Rivista del Nuovo Cimento, II, 253

Hauser, M. G., 1993, in Back to the Galaxy, AIP Conf. Proc. 278, ed. S. S. Holt \& F. Verter (New York: AIP), 201

Hauser, M. G., 1995, in Extragalactic Background Radiation, Space Telescope Sci. Inst. Symp. Ser. 7, ed. D. Calzetti, M. Livio, \& P. Madau, (Cambridge: Cambridge Univ. Press), 135

Hauser, M. G., et al. , 1991, in After the First Three Minutes, AIP Conf. Proc. 222, ed. S. Holt, C. L. Bennett, \& V. Trimble (New York: AIP), 161

Jahoda, K., Lockman, F. J., \& McCammon, D., 1990, ApJ, 354, 184

Kaufman, M., 1976, ApSpSci, 40, 369

Kawada, M., et al. , 1994, ApJ, 425, L89

Kelsall, T., et al. , 1993, Proc. SPIE Conf. 2019, Infrared Spaceborne Remote Sensing, ed. M. S. Scholl (Bellingham: SPIE), 190

Lockman, F. J., Jahoda, K., \& McCammon, D., 1986, ApJ, 302, 432

Low, F. J. \& Tucker, W. H., 1968, PRL, 22, 1538

Low, F. J. et al. , 1984, $A p J, 278, \mathrm{~L} 19$

Magner, T. J., 1987, OptEng, 26, 264

Mather, J.C., et al. , 1994, $A p J, 420,439$

Matsumoto, T., 1990, in The Galactic and Extragalactic Background Radiation, IAU Symposium 139, ed. S. Bowyer and C. Leinert

(Dordrecht: Kluwer), 317

Matsumoto, T., Akiba, M., \& Murakami, H., 1988, ApJ, 332, 575

Noda, M. et al. , 1992, ApJ, 391, 456

Partridge, R. B. \& Peebles, P. J. E., 1967, ApJ, 148, 377

Peebles, P. J. E., 1969, Phil. Trans. Royal Soc. London, A, 264, 279

Reach, W. T. et al. , 1995, Nature, to be published

Silverberg, R. F. et al. , 1993, Proc. SPIE Conf. 2019, Infrared Spaceborne Remote Sensing, ed. M. S. Scholl (Bellingham: SPIE), 180

Spiesman, W. J. et al. , 1995, $A p J, 442$, to be published

Stark, A. A. et al. , 1992, ApJS, 79, 77

Wainscoat, R. J. et al. , 1992, ApJS, 83, 111

Wheelock, S. L. et al. , 1994, IRAS Sky Survey Atlas Explanatory Supplement, JPL Pub. 94-11, (Pasadena: JPL), Appendix G

Wright, E.L. et al. , 1994, ApJ, 420, 450 